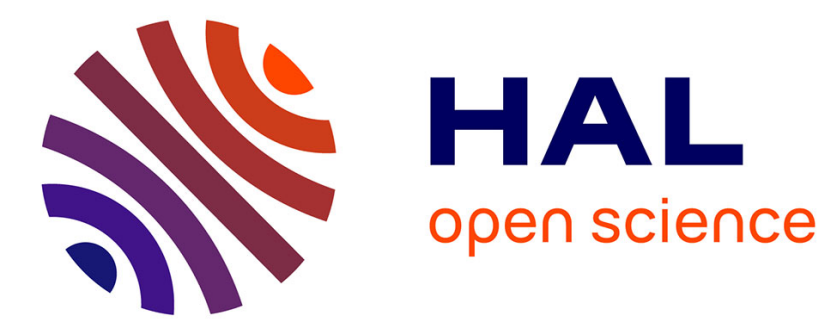

\title{
La neurologie environnementale
}

\author{
J. Reis
}

\section{To cite this version:}

J. Reis. La neurologie environnementale. Pratique Neurologique - FMC, 2019, 10, pp.1 - 8. 10.1016/j.praneu.2019.01.003 . hal-03486505

\section{HAL Id: hal-03486505 \\ https://hal.science/hal-03486505}

Submitted on 20 Dec 2021

HAL is a multi-disciplinary open access archive for the deposit and dissemination of scientific research documents, whether they are published or not. The documents may come from teaching and research institutions in France or abroad, or from public or private research centers.
L'archive ouverte pluridisciplinaire HAL, est destinée au dépôt et à la diffusion de documents scientifiques de niveau recherche, publiés ou non, émanant des établissements d'enseignement et de recherche français ou étrangers, des laboratoires publics ou privés.

\section{다)(1) $(5$}

Distributed under a Creative Commons Attribution - NonCommercial| 4.0 International 
Version of Record: https://www.sciencedirect.com/science/article/pii/S1878776219300032

Manuscript_df0623b6bd43d7ae2caad136344b9542

\title{
La Neurologie environnementale
}

\section{Environnemental Neurology}

\author{
Jacques Reis ${ }^{1,2 *}$
}

${ }^{1}$ Prof. Conv. Université de Strasbourg, Service de Neurologie (Prof Tranchant) . 1 Avenue Molière, 67200 Strasbourg

${ }^{2}$ Association RISE 3 rue du loir 67205 Oberhausbergen,

${ }^{*}$ Auteur correspondant :

Prof. Conv. Université de Strasbourg, Service de Neurologie (Prof Tranchant) . 1 Avenue Molière, 67200 Strasbourg

jacques.reis@wanadoo.fr

jacques.reis@unistra.fr 


\section{Résumé.}

La Neurologie environnementale est une approche nouvelle des pathologies neurologiques qui prend en compte l'impact de l'environnement dans leur genèse. Son champ d'intérêt recouvre de multiples disciplines qui s'occupent d'aspects spécifiques de notre environnement. Elle est basée sur une lecture à la fois analytique et globalisante des facteurs de risque environnementaux. Combinant les apports cliniques (histoire naturelle et évolutive de la maladie), à l'épidémiologie (notamment la génétique épidémiologique), à l'exposologie et aux modèles animaux, elle propose des modalités thérapeutiques renouvelées.

Mots clés : Environnement, facteur de risque, histoire naturelle, exposition, épidémiologie génétique

\section{Abstract}

Searching the environmental causes of the neurological diseases is one of the goals of Environmental Neurology (EN). A short historical background allows situating this new field. After having defined and described the environment, this article points to the interest of gathering separated fields dealing with specific environmental aspects. Then it shows how clinical study, epidemiology, exposology and basic research help to assess and establish the impact of environmental factors on the brain. EN provides also the bases for new or renewed therapeutic ways in neurology.

Key words: Environment, risk factor, natural history, exposition, genetic epidemiology 


\section{L'héritière d'une longue histoire?}

Il est habituel, quand on parle du rôle de l'Environnement dans la genèse d'une maladie, de rappeler que ce rôle a été reconnu dès l'antiquité. Néanmoins, il est très probable que le célèbre traité d'Hippocrate (1) décrivait des éléments avec des concepts bien différents d'aujourd'hui. En effet la nature des facteurs environnementaux a souvent été mystérieuse et a pu conduire à des confusions. La nature infectieuse des «fièvres palustres », attribuée à un air de mauvaise qualité au Moyen-Age, la malaria, n'est devenue évidente qu'après les découvertes du XIX et XXème siècles (anophèle et plasmodium), alors que l'effet bénéfique de la quinquina avait déjà été reconnu (2). L'effet délétère de l'air ambiant de Londres a constitué une menace dès le Moyen-Age (3). Le Grand Smog de Londres en 1952 n'était donc pas un accident imprévisible. La science des poisons et de leurs antidotes, illustrée par le traité des poisons de Maimonide (4) nous montre aussi que les toxiques et toxines ont de tout temps constitué une préoccupation majeure (5). Elle n'était pas encore « environnementale»!

C'est au cours du XIXe siècle que le mot d'origine française «environnement»va progressivement voir son usage se répandre aux USA et sa signification évoluer vers son sens actuel. Celui-ci n'apparait dans la langue française que vers les années 1960 (6). La prise de conscience « environnementaliste » internationale (initialement occidentale) est donc récente. Elle est liée à la perception d'une dégradation de l'environnement, amplifiée par les nombreuses catastrophes qui ont émaillé le XXème siècle. Prenant le relais de lanceurs d'alerte, des mouvements « écologistes » protestataires ont acquis rapidement une dimension politique. De manière parallèle, le champ scientifique et médical a commencé à prendre en compte l'environnement. A la suite de l'Hygiène, de la Santé Publique et de la Médecine tropicale, certaines spécialités médicales découvraient elles aussi l'Environnement.

En France c'est en 2002 que la Neurologie Environnementale nait lors des Journées de Neurologie de Langue Française à Tours avec l'organisation du Club de Neurologie (7). La Neurologie Française fut la première spécialité à reconnaître ce nouveau champ d'activité, première parmi les autres spécialités en France mais aussi au niveau international.

Vers une nouvelle approche de la maladie 
Proposer une nouvelle approche est une démarche heuristique qui déclenche de nombreuses interrogations (8). Qu'est-ce que l'Environnement ? comment procède-t-elle ? Que va-t-elle changer? Quel est son intérêt thérapeutique?

\section{Facteurs d'environnement}

Trop souvent les mots «facteurs d'environnement » apparaissent dans des articles sans qu'ils soient précisément définis. Voici la brillante définition d'Albert Einstein de l'Environnement : «The environment is everything that isn't me". Cependant cette définition est peu opérante ; une approche analytique de l'Environnement s'impose ; elle seule permet de répondre à la problématique de la causalité, du diagnostic étiologique. Le «tout» (l'environnement) est décomposé en «parties » (les facteurs d'environnement). Ces facteurs sont classés selon de multiples axes: origine, (technologique c'est-à-dire humaine versus naturel), nature (physique, chimique, biologique, facteur humain), milieu (eau, air, sol), cadre de vie (local confiné/ouvert, professionnel/domestique), biotope (rural/urbain, extrême par le climat, l'altitude), impact potentiel (danger/ innocuité) et complexité (alimentation, comportement social collectif/ individuel, interaction). Se manifestant dans un espace - temps précis, ils sont représentables dans un système à deux dimensions, le temps (durée) et le niveau d'action (local, régional, planétaire). Cette représentation bidimensionnelle est très utile pour caractériser les problèmes environnementaux et leurs enjeux, notamment sanitaires (figure 1). Elle illustre aussi leurs imbrications et interactions, montrant un des aspects de la complexité de l'Environnement.

\section{Approche globalisante}

L'approche globalisante de la Neurologie Environnementale propose d'inclure dans son champ d'activité toutes les disciplines médicales, touchant le système nerveux et qui abordent un aspect spécifique de notre environnement; c'est le cas, par exemple de la Neurologie Tropicale (Environnement géographique et climatique particulier), de la Neurotoxicologie (toxiques et toxines), de la Neuro-infectiologie et de la Médecine des environnements extrêmes (subaquatique, altitude, espace), entre autre. C'est nous semble-t-il un des intérêts majeurs de cette approche qui prend en compte l'environnement comme un tout, complexe. Ainsi les questions qu'elle soulève, nécessitent évidemment la collaboration avec les autres sciences du vivant (physiologistes, nutritionnistes, généticiens, vétérinaires, géographes de la Santé, etc.) et des sciences de la Terre (climatologues, géologues par exemple). 


\section{Démarche thérapeutique}

La démarche thérapeutique de la Neurologie environnementale est d'abord préventive. L'identification de facteurs de risques environnementaux doit conduire à leur éviction, évitement ou atténuation. Ces objectifs thérapeutiques sont nouveaux pour nous. Pourtant ils s'inscrivent dans la démarche médicale actuelle : mieux prévenir que «guérir ». En considérant les mécanismes adaptatifs, nous pourrions aussi agir en essayant d'atténuer l'impact délétère des facteurs non modifiables, par ceux qui le sont, notre mode de vie, la nutrition (alicaments), l'exercice physique et intellectuel et peut-être par de nouveaux médicaments « environnemento-mimétiques » (9).

\section{La part environnementale des maladies neurologiques.}

La démarche étiologique consiste à mettre en évidence et démontrer un lien de causalité entre un facteur (d'environnement) et une pathologie donnée.

\section{Ce qu'apporte le diagnostic clinique.}

\section{Classement}

Classer selon la nature de l'agression est un exercice inhabituel pour un clinicien, mais au combien utile. Voici l'exemple des syndromes parkinsoniens classés en fonction de la nature de l'agent causal. Les agressions de nature physique peuvent entrainer des syndromes parkinsoniens. C'est le cas des électrisations, des traumatismes crâniens liés à la boxe (10). Rappelons qu'un traumatisme crânien suite à une chute durant la phase inaugurale d'une maladie de Parkinson, est plutôt considéré comme symptôme que facteur de risque de la maladie, même si cette interprétation a fait débat (11). Les agressions biologiques sont infectieuses et toxiniques. Si les piqûres d'hyménoptères (guêpes) constituent des cas exceptionnels, les complications et séquelles neurologiques des infections sont des cas d'école. L'encéphalite léthargique a été décrite dans la suite de l'épidémie de grippe espagnole. Les signes extrapyramidaux sont classiques au cours des maladies à prion (10). Les agressions chimiques (iatrogènes et toxiques) constituent les modèles « expérimentaux » les plus fréquents de la maladie de Parkinson. Les neuroleptiques ont par définition un effet extrapyramidal. La liste des substances chimiques impactant le système extrapyramidal est particulièrement longue ; y figurent l'intoxication au monoxyde de carbone, au manganèse, au MPTP, aux organophosphorés, au trichloréthylène, aux solvants, (12-16) et, plus récemment, la pollution de l'air (17). 
Les syndromes extrapyramidaux, décrits en Guadeloupe et dans le Pacifique (Guam, Irian Jaya et Japon), liés à la consommation et/ou l'utilisation médicinale de certaines plantes ont un intérêt scientifique majeur pour la compréhension des maladies neurodégénératives et leurs relations. La tauopathie exotique de la Guadeloupe est liée à la consommation de fruits et de feuilles (décoctions) du corossolier contenant des neurotoxiques (isoquinolines et acétogénines) (18). Le syndrome Sclérose Latérale Amyotrophique-Complexe Parkinson Démence de Guam (SLA-CPD), appelé Muro au Japon (19) est une polyprotéinopathie induite par l'utilisation des fruits de cicas (alimentaire, usage chamanique) et/ou la consommation de chauves-souris (qui consommant ces fruits produisent une bioamplification des toxines) (20).

\section{Histoire naturelle}

L'histoire naturelle d'une affection décrit, entre autres, son évolution temporelle et sa distribution spatiale (géographique). Elle apporte ainsi des indications précieuses quant à sa cause. La distribution spatiale peut montrer une inégalité de répartition et des foyers. L'inégalité de répartition, par exemple de la sclérose en plaques (21-22) a stimulé la recherche causale : distribution liée à la latitude (21), rôle des migrations des Vikings (23). L'étude d'affections localisées, par exemple les cas japonais de SMON (Subacute MyeloOptico-Neuropathy) (24), de la maladie du Yusho (riz empoisonné par des PCB) (25) et de la maladie de Minamata (du nom de la baie où survint une intoxication par du méthylmercure) (26) peut orienter vers des causes génétiques, toxiques et environnementales. La courbe de l'évolution temporelle des cas incidents ou courbe épidémique signe typiquement une origine infectieuse mais aussi toxique ! C'est par exemple le cas de l'épidémie d'Encéphalopathie Spongiforme Bovine de 1998 (27), mais aussi des décès survenus en décembre 1952 durant le Smog de Londres (28), des cas de neuropathie optique cubaine survenue en 1999 (29), des épisodes de lathyrisme (30) et Konzo (31) provoqués par des famines survenues en Asie et Afrique. La disparition d'une maladie, son apparition brutale, tout comme l'augmentation de son incidence signent souvent des causes exogènes, environnementales. Le lathyrisme a sévi durant les périodes de guerres du XXème siècle en Europe dans les camps de prisonniers en raison de la malnutrition et/ou famine (30). Le Kuru, dont le dernier cas a été détecté en 1998, a maintenant disparu (32). L'explication retenue est l'interdiction en 1957 du cannibalisme rituel en Papouasie-Nouvelle Guinée, associée à une acculturation de la société Foré. Ces changements sociologiques drastiques ont précédé la reconnaissance scientifique du mécanisme du prion. Un autre foyer d'une maladie rare et négligée, déjà évoquée, le SLA- 
CPD dénommé Lytico-bodig en langue Chamorros, est en voie de disparition à Guam et dans la péninsule de Kii (Japon) sans que l'énigme soit totalement résolue (20).

\section{Ce qu'apporte l'Epidémiologie.}

\section{Epidémiologie}

L'épidémiologie s'est enrichie de l'apport du traitement de données massives («big data») qui permet, encore mieux, la comparaison entre régions ou pays, interrogeant les raisons des différences et inégalités des risques (33). Cette méthode, appliquée aux facteurs de risque des accidents vasculaires cérébraux (AVC), a permis de mettre en évidence le poids d'un risque longtemps méconnu : la pollution de l'air. Celle-ci explique, en moyenne, de 29,2\% des AVC ischémiques, avec une importance majeure dans les pays en voies de développement (34). Contributrice majeure (avec une autre cause environnementale, le plomb), la pollution de l'air est un facteur de risque d'une nouvelle catégorie, car sa prévention ne relève plus de l'individu mais de la régulation sociétale, donc politique.

\section{Génétique épidémiologique}

La génétique épidémiologique (35) étudie notamment les jumeaux et le milieu familial (fratrie, enfants adoptés, familles recomposées, cas conjugaux). Cette méthode détermine la part génétique (y compris la susceptibilité et l'effet maternel) et fournit à contrario une appréciation quantitative de la part non-génétique d'une maladie, donc indirectement du risque environnemental (Sadovnick D. L'épidémiologie Génétique : indications pour les rôles respectifs des gènes et de l'environnement dans l'étiologie de la SEP. Présentation JNLF Marseille CNE 28-04-2005). Elle est particulièrement pertinente pour les maladies complexes impliquant l'environnement. Voici l'exemple de la sclérose en plaques. Le risque relatif (RR) de récurrence concerne l'agrégat familial et évalue la probabilité pour un parent du propositus (frère, cousin, etc.) d'être affecté par la même maladie (36). Pour la sclérose en plaques, un effet «familial» est clairement mis en évidence ; dans une même fratrie le risque (vie entière) est de $3.5 \%$, comparé au RR dans la population générale (2/1000) ; ce RR est donc multiplié par 15 à 25 ! (36-38)

Les études de jumeaux mono- et dizygotes donnent un ordre de grandeur de la part génétique entre jumeaux qui est de 1 à 4/1000. La probabilité du co-jumeau «non affecté » de développer la maladie est mesurée par le taux de concordance : les deux vont développer la maladie (dans laps de temps défini). Il est de 3 à $5 \%$ pour les jumeaux dizygotes (DZ) et de 24 à 35\% pour les jumeaux monozygotes (MZ) (36). Une méta-analyse utilisant des méthodes 
statistiques particulières a montré un taux d'héritabilité de $50 \%$ et une corrélation de $42 \%$ pour les jumeaux DZ (39). Les GWAS (Genome-Wide Association Studies) donnent des résultats comparables : la part héritable est de près de $50 \%$ (40).

\section{Part environnementale de la SEP}

De manière plus classique, les études de migrations et de l'évolution du sex-ratio illustrent qualitativement la part environnementale de la SEP. Lors d'une migration, les acquis génétiques et épigénétiques sont soumis à un nouvel environnement ; le risque de développer une SEP est alors fonction de l'âge de départ (avant ou après 15 ans) et du type de migration, d'un pays à haut risque élevé vers un pays à faible risque (et prévalence) et vice-versa (4143). Ainsi les migrants de plus de 15 ans se déplaçant d'une zone à haut risque vers une zone à faible risque, gardent le risque de leur terre natale (44). Ceci est un des arguments qui a conduit Kurtzke à considérer non seulement que la SEP est une maladie acquise au début de l'adolescence mais aussi quelle est de nature infectieuse (21). Cette part environnementale est aussi envisagée pour expliquer l'évolution du sex-ratio. Un décalage progressif du rapport de $2 \mathrm{~F} / 1 \mathrm{H}$ vers un ratio de $3 \mathrm{~F} / 1 \mathrm{H}$ a été démontré. Ce glissement n'est pas lié à une cause génétique. Mais aucune hypothèse explicative satisfaisante n'a été proposée à ce jour (45-47). La conclusion est claire : l'environnement est un facteur contributif majeur, la part génétique estimée variant entre $30 \%$ à $50 \%$ (48).

\section{Causes environnementales}

L'épidémiologie génétique quantifie aussi indirectement la place des causes environnementales dans les maladies neurodégénératives. Selon les critères de diagnostic retenus (cliniques uniquement ou associés au PET-scan), la part génétique de la maladie de Parkinson (chez les jumeaux monozygotes, après 50 ans) varie de $16 \%$ (49) à $75 \%(49,50)$.

Bien que l'héritabilité de la maladie d'Alzheimer soit importante (79\%) avec des taux de concordance pour des jumeaux MZ variant de 44\% à 83\% (51, 52), l'utilisation du PET scan (11C-PiB), en montrant une dissociation entre les performances cognitives et la fixation par le biomarqueur des dépôts amyloïdes, souligne le facteur environnemental (53).

\section{Ce qu'apportent l'exposologie et la neurotoxicologie.}

Elles permettent de poser des questions cruciales. Quels sont les milieux (air, eau, alimentation) et vecteurs d'exposition qui nous mettent en contact avec des agresseurs 
potentiels de l'environnement? Comment ces agresseurs pénètrent-ils dans l'organisme (épithélium cutané, nasal, pulmonaire et digestif) et ciblent-ils le système nerveux (action directe) ? A quelle période de la vie sommes-nous exposés ? Quel est l'effet d'une exposition permanente? Quels sont les moments critiques (fenêtres critiques) durant lesquels notre organisme est le plus vulnérable $(54,55)$ ?

\section{Exposition aux facteurs de risque}

L'approche systématique de l'exposition aux facteurs de risque, en fonction de critères multiples, vecteurs et agents, espaces de survenue(localisés/planétaires), temporalité (durée d'exposition) permet la mise en évidence d'interactions. Voici l'exemple de la SEP ; on constate que des facteurs de risques classiques sont impactés aussi par la qualité de l'air ambiant (tableau 1).

\section{Systèmes de régulation}

Les sciences fondamentales montrent que l'organe critique qu'est le cerveau, est protégé par plusieurs systèmes de régulation (régulation de la vasomotricité, barrière hémato-méningée, système microglial). Il reste accessible par voie systémique aux messagers (hormones, cytokines) produits par les organes périphériques (y compris poumons et intestins) (56). La voie nasale, qui le met en contact avec l'environnement revêt une importance particulière. Les études récentes questionnent son rôle dans la pathogénie de nombreuses affections neurologiques $(57,58)$. Le passage de germes (virus, parasites, bactéries) est connu de longue date (59). Plusieurs médicaments sont à administration nasale (60). Les particules fines et nanoparticules pénètreraient aussi par voie nasale et atteindraient le bulbe olfactif, comme ceci a été démontré pour le manganèse $(61,62)$. La pénétration de toxines par voie nasale est proposée dans la physiopathogénie de la sclérose en plaques $(63,64)$.

\section{Début des maladies neurodégénératives}

La question majeure du début des maladies neurodégénératives est récurrente : quand et comment débutent-elles ? Prenons l'exemple de la maladie d'Alzheimer. Même si les études cliniques ont permis d'identifier les symptômes précoces avant le diagnostic de démence $(65,66)$, corroborés par le staging anatomo-pathologique $(67,68)$, ce sont les modèles animaux qui sont les plus informatifs. C'est le cas des modèles de maladie d'Alzheimer développés chez les rongeurs et le singe. Ainsi une exposition périnatale au plomb a un effet double, immédiat (élévation des biomarqueurs de dégénérescence qui disparaît à l'arrêt de 
l'exposition) et retardé. Lors de la sénescence, cette élévation réapparait spontanément, signant la reprise du processus dégénératif $(69,70)$. Ces modèles permettent de mieux évaluer le risque majeur que constitue la pollution de l'air ambiant. En effet des études épidémiologiques et anatomopathologiques menées dans la ville de Mexico montrent les effets délétères de l'air pollué chez les enfants $(71,72)$, au point de faire la une aux USA de journaux "grand public" (73). La crainte est de préparer une explosion des syndromes démentiels précoces dans les mégapoles polluées.

\section{Quelles perspectives thérapeutiques?}

\section{Prévention}

La prévention reste le maitre mot. La connaissance précise des risques environnementaux auxquels nous sommes exposés, quel que soit le lieu et moment où ils surviennent (travail, domicile, loisirs), permet de les éviter, les réduire et/ou gérer.

La reconnaissance professionnelle de la maladie de Parkinson (MP n 58 , décret du 4 mai 2012) est une opportunité pour occuper un champ d'activité dont nous sommes habituellement éloignés. Le neurologue devrait participer activement à l'identification du facteur de risque potentiel professionnel (agriculteurs, exposition à des métaux dits «lourds »), à l'évaluation du facteur toxique, à l'argumentation causale pour déclarer la MP $(74,75)$. Quand les risques sont discutés, la problématique devient sociétale et la prise de décision est politique. Rappelons les conflits d'intérêts, de notoriété publique, engendrés par le pesticide le plus utilisé au monde, le glyphosate. Ce cancérigène avéré est aussi un neurotoxique et un perturbateur endocrinien $(76,77)$. Le doute est-il encore permis ?

\section{Traitements}

De quels traitements disposons pour gérer les maladies induites en dehors des médications symptomatiques, quand les risques sont inévitables ? Nous sommes restés des utilisateurs prudents des chélateurs des métaux dans des indications précises (neutoxicologie, maladie de surcharge) même si de nouvelles options (chélateur du fer) apparaissent $(78,79)$. Il ne s'agit en aucun cas de «médecines alternatives »! Ces dernières proposent des traitements de détoxification en réponse aux inquiétudes nées de la prise de conscience de la contamination chimique globale, elle, bien réelle. Ceux-ci ne sont ni validées ni dépouvus d'effets secondaires (peu documentés) (80). Dans une optique de neuroprotection, des médicaments sont développés ou repositionnés tels que des antagonistes du glutamate, des antioxydants, 
des facteurs trophiques (81). La recherche de médicaments «environnemento-mimétiques » apparaît comme une voie théoriquement prometteuse, bien qu'encore expérimentale $(9,82)$.

\section{Approches non médicamenteuses}

Les approches non médicamenteuses sont connues. Si l'entrainement cognitif nous est familier, son efficacité réelle reste discutée $(83,84)$. Les effets bénéfiques de l'exercice physique sont bien documentés (85-87). La question d'un effet bénéfique de l'exercice effectué en milieu naturel et/en extérieur est aussi posée (88). C'est certainement cette démarche de « resourcement dans et par la nature » qui a conduit à une discipline méconnue en Occident, la médecine forestière qui s'est développée au Japon pour se propager en Corée et Chine $(89,90)$. L'effet d'un environnement perçu comme sain devient ainsi une approche nouvelle du «bien-être » et un effet (antistress) objectivable (91). L'intérêt d'une nutrition de qualité et des alicaments nous amène à élargir nos compétences. Nous allons devoir mieux prendre en compte les compléments alimentaires (oligoéléments, iode, Oméga 3) et les vitamines (acide folique, Vitamines D et E) (92-94), conseiller des régimes (méditerranéen, cétogène, japonais, DASH) $(95,96)$, tout ceci en en fonction des pathologies présentées par nos patients (97-99).

\section{Conclusion.}

Considérer l'impact de l'Environnement en médecine, modifie la compréhension des maladies, notamment neurologiques. Que cet impact soit direct ou résultant d'une interaction avec les gènes et l'épigénome, il va modifier nos approches cliniques, épidémiologiques, physiopathologiques donc finalement thérapeutiques des maladies. Son étude constitue un nouveau challenge, comme l'a été l'apport de la génétique.

\section{Déclaration d'intérêts}

L'auteur déclare ne pas avoir de lien d'intérêts relatif à cet article. 


\section{Bibliographie}

1 Hippocrates, translation J. Jouhanna Tome II, 2ème partie, des eaux, des airs et des lieux. Edition Les belles lettres ; 2003, 452 p.

2 Rocco F. Traduit par Gamberini MC. L'Écorce miraculeuse. Le remède qui changea le monde. Les éditions noir sur blanc ; 2006, 336 p

3 Longhurst, J., Barnes, J., Bushel, C., Chatterton, T., Hayes, E. T., Prestwood, E. et al. (2016) What has been achieved in the 60 years since the first Clean Air Act? In: Routes to Clean Air 2, Bristol, UK, 11-12 October 2016. Available from: http://eprints.uwe.ac.uk/30197 4 Maimonides M. editor Muntner S. ITRI. Treatise on Poisons and Their Antidotes. J. B. Philadelphia Lippincott ; 1966, 77p

5 Birlouez E. Histoire des poisons, des empoisonnements et des empoisonneuses. Rennes :Editions Ouest-France ; 2016, 127 p.

6 Rey A. Dictionnaire historique de la langue française, 3 vol. Le Robert, 2006.

7 Reis J. Maladies neurologiques et environnement. Environ Risque et Santé. 2014; 13 (6) : $177-9$.

8 Reis J. \& Romàn GC. Environmental Neurology: a promising a new field of practice and research. J. Neurol. Sci. 2007;262(1-2):3-6.

9 McOmish CE \& Hannan AJ. Enviromimetics: exploring gene - environment interactions to identify therapeutic targets for brain disorders. Expert Opin. Ther. Targets $2007 ; 11(7)$ : 899-913

10 Tison F. Maladie de Parkinson et syndromes parkinsoniens. In Rascol A. La maladie de Parkinson. Paris: Masson; 1998 : pp 41-58.

11 Camacho-Soto A, Warden MN, Searles Nielsen S, Salter A, Brody DL, Prather H et al. Traumatic brain injury in the prodromal period of Parkinson's disease: A large epidemiological study using medicare data. Ann Neurol. 2017; 82(5):744-754.

12 Spencer P.S. Environmental Agents and Parkinson's disease. New York M Dekker; 1995: pp 319-365.

13 Gash DM, Rutland K, Hudson NL, et al Trichloroethylene: Parkinsonism and complex 1 mitochondrial neurotoxicity. Ann Neurol. 2008 ;63:184-92.

14 Reis J, Benbrick E, Bonneterre V, Spencer PS. Parkinson's disease and solvents: Is there a causal link? Rev. Neurol . 2016 ;172 : 761-765.

15 Shin HW, Chung SJ. Drug-Induced Parkinsonism J Clin Neurol. 2012; 8:15-21. 
16 Bhatt MH, Elias MA, Mankodi AK. Acute and reversible parkinsonism due to organophosphate pesticide intoxication: five cases. Neurology $1999 ; 52: 1467-71$

17 Ritz B, Lee P-C, Hansen J et al; Traffic-Related Air Pollution and Parkinson's disease in Denmark: A Case-Control Study. Environ Health Perspect. 2016; 124(3): 351-356.

18 Caparros-Lefebvre D, Sergeant N, Lees A, et al. Guadeloupean parkinsonism: a cluster of progressive supranuclear palsy-like tauopathy. Brain. 2002; 125: 801-11.

19 Kihira T, Yoshida S, Kondo T. et al. An increase in ALS incidence on the Kii Peninsula, 1960-2009: a possible link to change in drinking water source. Amyotroph Lateral Scler. 2012;13(4):347-50

20 Spencer PS, Palmer VS, Kisby GE. Seeking environmental causes of neurodegenerative disease and envisioning primary prevention. Neurotoxicology. 2016;56:269-283.

21 Kurtzke JF. Epidemiologic evidence for multiple sclerosis as an infection. Clin Microbiol Rev. 1993;6(4):382-427.

22 Pugliatti M, Sotgiu S, Rosati G. The worldwide prevalence of multiple sclerosis. Clin Neurol Neurosurg. 2002;104(3):182-91.

23 Poser CM. The dissemination of multiple sclerosis: a Viking saga? A historical essay. Ann Neurol. 1994; 36 Suppl 2: S231-43.

24 Konagaya M. SMON: toxicity of clioquinol and the status quo. Brain Nerve. 2015; 67(1): 49-62.

25 Reggiani G, Bruppacher R. Symptoms, signs and findings in humans exposed to PCBs and their derivatives. Environ Health Perspect. 1985; 60: 225-32.

26 Grandjean P, Satoh H, Murata K, Eto K. Adverse effects of methylmercury: environmental health research implications. Environ Health Perspect. 2010; 118(8): 1137-45.

27 Brugère-Picoux J. L'encéphalopathie spongiforme bovine, Où en sommes-nous après les crises de 1996 et de 2000 ? Association française pour l'information scientifique 2011; 292 : 1-12. http://www.pseudo-sciences.org consulté le 20.04.2018

28 Bell ML., Davis DL. Reassessment of the Lethal London Fog of 1952: Novel Indicators of Acute and Chronic Consequences of Acute Exposure to Air Pollution. Environ Health Perspect. 2001; 109(suppl 3):389-394.

29 Román GC. An epidemic in Cuba of optic neuropathy, sensorineural deafness, peripheral sensory neuropathy and dorsolateral myeloneuropathy. J Neurol Sci. 1994; 127(1): 11-28.

30 Reis J, Spencer P.S. Lathyrism. In: Chopra J. S., Sawhney I.M.S. Neurology in Tropics. 2nd ed. New Delhi: Elsevier India; 2015 : pp 369-78. 
31 Tylleskär T., Tshala-Katumbay D. Konzo: a permanent, non-progressive motor neuron disorder. In: Chopra J. S., Sawhney I.M.S. Neurology in Tropics. 2nd ed. New Delhi: Elsevier India; 2015 : pp 379- 388.

32 Collinge J., Alpers MP. Introduction. Philos Trans R Soc Lond B Biol Sci. 2008; 363(1510): 3607-3612.

33 Mooney S.J, Westreich D.J, El-Sayed A.M. Epidemiology in the Era of Big Data. Epidemiology. 2015; 26: 390-394.

34 Feigin VL, Roth GA, Naghavi M. et al. Global burden of stroke and risk factors in 188 countries, during 1990-2013: a systematic analysis for the Global Burden of Disease Study 2013. Lancet Neurol. 2016 ; 15: 913-24

35 Dorak T. Genetic Epidemiology. http://www.dorak.info/epi/genetepi.html Access on 20.04.2018

36 Ebers G. Interactions of environment and genes in multiple sclerosis. J Neurol Sci. 2013; 334(1-2):161-3.

37 Dyment DA, Ebers GC, and Sadovnick AD. Genetics of multiple sclerosis. Lancet Neurol. 2004; 3:104-110

38 Willer C. J. , Dyment D. A., Risch N. J., Sadovnick A. D. , Ebers G. C. and CCSG. Twin concordance and sibling recurrence rates in multiple sclerosis. PNAS $2003 ; 100: 12877-$ 12882.

39 Fagnani C, Neale MC, Nistico L, Stazi MA, Ricigliano VA, Buscarinu MC, et al.

Twin studies in multiple sclerosis: a meta-estimation of heritability and environmentality. Mult Scler. 2015 ; 21(11):1404-13.

40 Parnell GP., Booth DR. The Multiple Sclerosis (MS) Genetic Risk Factors Indicate both acquired and Innate Immune cell Subsets Contribute to MS. Pathogenesis and identify novel therapeutic opportunities. Front. Immunol. 2017; 8: 425.

41 Dean G, Elian M. Age at immigration to England of Asian and Carribean immigrants and the risk of developing multiple sclerosis. J Neurol Neurosurg Psychiatry 1997; 63: 565-8.

42 Dean G, Kurtzke JF. On the risk of multiple sclerosis according to age at immigration to South Africa. Br Med J 1971; 3: 725-9.

43 Cabre P, Signate A, Olindo S, Merle H, Caparros-Lefebvre D, Béra O. et al.

Role of return migration in the emergence of multiple sclerosis in the French West Indies. Brain 2005 ; 128 : 2899-2910. 
44 Kurtzke JF. Epidemiology in multiple sclerosis: a pilgrim's progress. Brain. 2013;136 : 2904-17.

45 Trojano M, Lucchese G, Graziano G, Taylor BV, Simpson S Jr, Lepore V. et al. Geographical Variations in Sex Ratio Trends over Time in Multiple Sclerosis. PLoS ONE 2012; 7(10): e48078

46 Disanto G, Ramagopalan SV. On the sex ratio of multiple sclerosis. Mult Scler. 2013;19:34.

47 Boström I Landtblom A-M Does the changing sex ratio of multiple sclerosis give opportunities for intervention? Acta Neurol. Scand. 2015; 132: 42-45.

48 Olsson T, Barcellos LF, Alfredsson L. Interactions between genetic, lifestyle and environmental risk factors for multiple sclerosis. Nat Rev Neurol. 2017 ;13(1):25-36.

49 Tanner CM, Ottman R, Goldman SM, Ellenberg J, Chan P, Mayeux R, et al. Parkinson disease in twins. An etiologic study JAMA $1999 ; 281: 341-6$

50 Piccini P, Burn DJ, Ceravolo R, Maraganore D, Brooks DJ. The role of inheritance in sporadic Parkinson's disease: evidence from a longitudinal study of dopaminergic function in twins. Ann Neurol 1999;45:577-82

51 Gatz M, Pedersen N.L. Study of Dementia in Swedish Twins. Twin Res Hum Genet. 2013; 16: 1-7.

52 Gatz M, Reynolds CA, Fratiglioni L, Johansson B, Mortimer JA, Berg S, et al. Role of Genes and Environments for Explaining Alzheimer Disease Arch. Gen Psychiatry. 2006;63:168-174

53 Scheinin N.M., Aalto S., Kaprio J., Koskenvuo M., Räihä I., Rokka J., et al. Early detection of Alzheimer disease 11C-PiB PET in twins discordant for cognitive impairment Neurol 2011; 77:453-60

54 Adams J, Barone Jr S., LaMantia A, Philen R, Rice DC, Spear L, et al. Workshop to Identify Critical Windows of Exposure for Children's Health: Neurobehavioral Work Group Summary. Environ Health Perspect. 2000 ; 108(suppl 3):535-544.

55 Meredith RM. Sensitive and critical periods during neurotypical and aberrant neurodevelopment: a framework for neurodevelopmental disorders. Neurosci Biobehav Rev. 2015;50:180-8.

56 Mumaw CL, Levesque S, McGraw C, Robertson S, Lucas S, Stafflinger JE, et al. Microglial priming through the lung-brain axis: the role of air pollution-induced circulating factors. FASEB J. 2016; 30:1880-91. 
57 Hawkes CH, Shephard BC, Daniel SE. Is Parkinson's disease a primary olfactory disorder? QJM. 1999; 92(8):473-80.

58 Tonelli LH, Postolache TT. Airborne inflammatory factors: "from the nose to the brain". Front Biosci (Schol Ed). 2010; 2: 135-52.

59 Harberts E, Yao K, Wohler JE, Maric D, Ohayon J, Henkin R, et al. Human herpesvirus-6 entry into the central nervous system through the olfactory pathway. Proc Natl Acad Sci U S A. $2011 ; 108: 13734-9$.

60 Pardeshi CV, Belgamwar VS. Direct nose to brain drug delivery via integrated nerve pathways bypassing the blood-brain barrier: an excellent platform for brain targeting.

Expert Opin Drug Deliv. 2013;10:957-72.

61 Lucchini R.G., Dorman D.C., Elder A., Veronesi B. Neurological impacts from inhalation of pollutants and the nose-brain connection. NeuroToxicology. $2012 ; 33$ : 838-841

62 Brenneman KA, Wong BA, Buccellato MA, Costa ER, Gross EA, Dorman DC. Direct olfactory transport of inhaled manganese $((54) \mathrm{MnCl}(2))$ to the rat brain: toxicokinetic investigations in a unilateral nasal occlusion model. Toxicol Appl Pharmacol. 2000; 169: 23848.

63 Gay F. Bacterial toxins and Multiple Sclerosis. J Neurol Sci. 2007; 262: 105-12.

64 Gay F. Nose to brain: Is the trigeminal nerve a conduit for CNS disease? Mult Scler Relat Disord. $2012 ; 1(4): 154-5$.

65 Gatz M, Reynolds CA, Finkel D, Pedersen NL, Walters E. Dementia in Swedish twins: predicting incident cases. Behav Genet. 2010; 40(6):768-75.

66 Devanand DP. Olfactory Identification Deficits, Cognitive Decline, and Dementia in Older Adults. Am J Geriatr Psychiatry. 2016; 24(12):1151-1157.

67 Delacourte A, Sergeant N, Wattez A, Maurage CA, Lebert F, Pasquier F, et al. Tau aggregation in the hippocampal formation: an ageing or a pathological process?

Exp Gerontol. 2002; 37(10-11):1291-6.

68 Braak E, Griffing K, Arai K, Bohl J, Bratzke H, Braak H. Neuropathology of Alzheimer's disease: what is new since A. Alzheimer? Eur Arch Psychiatry Clin Neurosci. 1999;249 Suppl $3: 14-22$.

69 Zawia NH., Basha MR. Environmental Risk Factors and the Developmental Basis for Alzheimer's Disease. Rev. Neurosci. 2005; 16: 325-337.

70 Lahiri DK, Maloney B, Zawia NH. The LEARn model: an epigenetic explanation for idiopathic neurobiological diseases. Mol Psychiatry. 2009 ;14:992-1003. 
71 Calderón-Garcidueñas L., Gónzalez-Maciel A., Reynoso-Robles R. et al. Hallmarks of Alzheimer disease are evolving relentlessly in Metropolitan Mexico City infants, children and young adults. APOE4 carriers have higher suicide risk and higher odds of reaching NFT stage $\mathrm{V}$ at $\leq 40$ years of age. Environmental Research 164 (2018) 475-487

72 Maher BA, Ahmed IA, Karloukovski V, MacLaren DA, Foulds PG, Allsop D, et al. Magnetite pollution nanoparticles in the human brain. Proc Natl Acad Sci U S A. 2016 ; 113(39):10797-801.

73 Dovey D. Alzheimer's disease starts in childhood, with symtoms found in babies less than a year old, study shows. 2018, access on the 20.04.2018 http://www.newsweek.com/airpollution-alzheimers-disease-children-891868

74 Elbaz A, Moisan F. The scientific bases to consider Parkinson's disease an occupational disease in agriculture professionals exposed to pesticides in France. J Epidemiol Community Health. $2016 ; 70: 319-21$.

75 Defebvre L. Maladie de Parkinson : rôle des facteurs génétiques et environnementaux. Implication en pratique clinique quotidienne Rev. Neurol. 2010; 166: 764-769

76 Myers JP, Antoniou MN, Blumberg B, Carroll L, Colborn T, Everett LG, et al. Concerns over use of glyphosate-based herbicides and risks associated with exposures: a consensus statement. Environ Health. 2016;15:19. pp13.

77 Kissane Z, Shephard JM. The rise of glyphosate and new opportunities for biosentinel early-warning studies. Conserv Biol. 2017;31:1293-1300.

78 Ward RJ, Dexter DT, Crichton RR. Neurodegenerative diseases and therapeutic strategies using iron chelators. J Trace Elem Med Biol. 2015;31:267-73.

79 Youdim MB, Stephenson G, Ben Shachar D. Ironing iron out in Parkinson's disease and other neurodegenerative diseases with iron chelators: a lesson from 6-hydroxydopamine and iron chelators, desferal and VK-28. Ann N Y Acad Sci. 2004; 1012: 306-25.

80 Allen J, Montalto M, Lovejoy J, Weber W. Detoxification in Naturopathic Medicine:

A Survey. J Altern Complement Med. 2011; 17:1175-80.

81 Vajda FJ. Neuroprotection and neurodegenerative disease. J Clin Neurosci. 2002 ; 9: 4-8.

82 Nithianantharajah J, Hannan AJ. Enriched environments, experience-dependent plasticity and disorders of the nervous system. Nat Rev Neurosci. 2006 ; 7: 697-709.

83 Bahar-Fuchs A, Clare L, Woods B. Cognitive training and cognitive rehabilitation for mild to moderate Alzheimer's disease and vascular dementia. Cochrane Database Syst Rev. 2013; (6): CD003260, pp 106. 
84 Kallio EL, Öhman H, Kautiainen H, Hietanen M, Pitkälä K. Cognitive Training Interventions for Patients with Alzheimer's Disease: A Systematic Review. J Alzheimers Dis. 2017; 56(4):1349-1372.

85 Ahlskog JE. Does vigorous exercise have a neuroprotective effect in Parkinson disease? Neurology. 2011;77(3):288-94.

86 Seifert T. Exercise and neurologic disease. Continuum (Minneap Minn). 2014; 20(6 Sports Neurology):1667-82.

87 Mayo Clinic Staff. Exercise: 7 benefits of regular physical activity. Access 05_05_2018 on https://www.mayoclinic.org/healthy-lifestyle/fitness/in-depth/exercise/art-20048389

88 Coon JT, Boddy K, Stein K, Whear R, Barton JR, Depledge MH. Does Participating in Physical Activity in Outdoor Natural Environments Have a Greater Effect on Physical and Mental Wellbeing than Physical Activity Indoors? A Systematic Review. Environ. Sci. Technol 2011; 45(5):1761-72.

89 Park BJ, Tsunetsugu Y, Kasetani T, Kagawa T, Miyazaki Y. The physiological effects of Shinrin-yoku (taking in the forest atmosphere or forest bathing): evidence from field experiments in 24 forests across Japan. Environ Health Prev Med. 2010;15(1):18-26.

90 Ochiai H, Ikei H, Song C, Kobayashi M, Takamatsu A, Miura T, Kagawa T, Li Q, Kumeda S, Imai M, Miyazaki Y. Physiological and psychological effects of forest therapy on middle-aged males with high-normal blood pressure. Int J Environ Res Public Health. 2015;12(3):2532-42.

91 Husk K, Lovell R, Cooper C, Stahl-Timmins W, Garside R. Participation in environmental enhancement and conservation activities for health and well-being in adults: a review of quantitative and qualitative evidence. Cochrane Database Syst Rev. 2016 May 21;(5):CD010351. pp 256.

92 Mowry EM, Pelletier D, Gao Z, Howell MD, Zamvil SS, Waubant E. Vitamin D in clinically isolated syndrome: evidence for possible neuroprotection. Eur J Neurol. 2016; 23: 327-32.

93 Venkatesan R, Ji E, Kim SY. Phytochemicals that regulate neurodegenerative disease by targeting neurotrophins: a comprehensive review. BioMed Research Inter. 2015. 2015: 814068 .

94 Subash S, Essa MM, Al-Adawi S, Memon M A., Manivasagam T, Akbar M. Neuroprotective effects of berry fruits on neurodegenerative diseases. Neural Regen Res. 2014; 9(16): 1557-1566. 
95 Estruch R, Ros E, Salas-Salvadó J, Covas MI, Corella D, Arós F, et al. PREDIMED Study Investigators. Primary prevention of cardiovascular disease with a Mediterranean diet. N Engl J Med. 2013; 368(14):1279-90.

96 Pinto A, Bonucci A, Maggi E, Corsi M, Businaro R. Anti-Oxidant and Anti-Inflammatory Activity of Ketogenic Diet: New Perspectives for Neuroprotection in Alzheimer's Disease. Antioxidants (Basel). $2018 ; 28 ; 7(5)$.

97 Lehert P, Villaseca P, Hogervorst E, Maki PM, Henderson VW. Individually modifiable risk factors to ameliorate cognitive aging: a systematic review and meta-analysis. Climacteric. 2015; 18(5):678-89.

98 Seidl SE, Santiago JA, Bilyk H. Potashkin JA. The emerging role of nutrition in Parkinson's disease. Front Aging Neurosci. 2014; 6: 36.

99 Sherzai AZ, Tagliati M, Park K, Pezeshkian S, Sherzai D. Micronutrients and Risk of Parkinson's Disease: A Systematic Review. Gerontol Geriatr Med. 2016;2: 1-11

100 Jin Y, de Pedro-Cuesta J, Söderström M, Stawiarz L, Link H. Seasonal patterns in optic neuritis and multiple sclerosis: a meta-analysis. J Neurol Sci. 2000;181(1-2):56-64.

102 Spelman T, Gray O, Trojano M, Petersen T, Izquierdo G, Lugaresi A, et al. Seasonal variation of relapse rate in multiple sclerosis is latitude dependent. Ann Neurol. 2014; 76(6): 880-90.

103 Giovannoni G, Ebers G. Multiple sclerosis: the environment and causation. Curr Opin Neurol. 2007; 20(3):261-8.

104 Tremlett H, van der Mei IA, Pittas F, Blizzard L, Paley G, Mesaros D, et al. Monthly ambient sunlight, infections and relapse rates in multiple sclerosis. Neuroepidemiology. 2008; 31(4):271-9.

105 Sibley WA, Bamford CR, Clark K. Clinical viral infections and multiple sclerosis. Lancet. 1985; 1(8441):1313-5.

106 Edwards S, Zvartau M, Clarke H, Irving W, Blumhardt LD. Clinical relapses and disease activity on magnetic resonance imaging associated with viral upper respiratory tract infections in multiple sclerosis. J Neurol Neurosurg Psychiatry. 1998; 64(6):736-41.

107 Ascherio A, Munger KL. Environmental risk factors for multiple sclerosis. Part I: the role of infection. Ann Neurol. 2007; 61(4):288-99.

108 Gay D, Dick G, Upton G. Multiple Sclerosis associated with sinusitis. A case controlled study in general practice. Lancet.1986; 1(8485): 815-9.

109 Calderón-Garcidueñas L, Leray E, Heydarpour P, Torres-Jardón R, Reis J. 
Air pollution, a rising environmental risk factor for cognition, neuroinflammation and neurodegeneration: The clinical impact on children and beyond. Rev Neurol (Paris). 2016; 172(1):69-80.

110 O'Gorman C, Lucas R, Taylor B. Environmental risk factors for multiple sclerosis: a review with a focus on molecular mechanisms. Int J Mol Sci. 2012; 13(9):11718-52.

111 Barragán-Martínez C, Speck-Hernández CA, Montoya-Ortiz G, Mantilla RD, Anaya JM, Rojas-Villarraga A. Organic Solvents as Risk Factor for Autoimmune Diseases: A Systematic Review and Meta-Analysis. PLoS One. 2012; 7(12): e51506.

112 Hedström AK, Katsoulis M, Hössjer O, Bomfim IL, Oturai A, Sondergaard HB, et al. The interaction between smoking and HLA genes in multiple sclerosis: replication and refinement. Eur J Epidemiol. 2017; 32(10):909-919. 
Table 1. Interrelations entre facteurs de risques connus et air ambiant dans la sclérose en plaque.

\begin{tabular}{|c|c|c|c|}
\hline Facteur & Effets & Effet de la pollution de l'air & références \\
\hline Saisonnalité & $\begin{array}{l}\text { Excès biphasique des poussées de } \\
\text { SEP au printemps et hiver }\end{array}$ & & $100-102$ \\
\hline Saisonnalité & $\begin{array}{l}\text { Recrudescence des IRVAS en } \\
\text { automne +hiver (infections respiratoires } \\
\text { voies aériennes supérieures) }\end{array}$ & $\begin{array}{l}\text { Susceptibilité mucosale aux } \\
\text { virus liée au froid (via une } \\
\text { baisse de l'immunité innée) }\end{array}$ & 102 \\
\hline Vitamine D & $\begin{array}{l}\text { Diminution du risque de poussées (taux } \\
\text { sériques élevés) }\end{array}$ & $\begin{array}{l}\text { Diminue les taux sériques de Vit } \\
\text { D }\end{array}$ & 103 \\
\hline Vitamine D & $\begin{array}{l}\text { Ascension taux sérique en été et chute } \\
\text { durant l'hiver }\end{array}$ & $\begin{array}{l}\text { Exposition maternelle à NO2 et } \\
\text { PM10 diminue taux } 25 \mathrm{OH} \text { CC à } \\
\text { la naissance }\end{array}$ & 103 \\
\hline Ultraviolet & $\begin{array}{l}\text { Association inverse, poussées/ UV+ Vit } \\
D+\text { ensoleillement mensuel }\end{array}$ & Diminue l'exposition aux UV & 104 \\
\hline IRVAS & Augmentation du RR (2.1) de poussées & $\begin{array}{l}\text { Susceptibilité accrue aux } \\
\text { infections liée aux PM10 }\end{array}$ & 105106 \\
\hline $\begin{array}{l}\text { Infection par } \\
\text { Epstein-Barr }\end{array}$ & $\begin{array}{l}\text { RR poussées: } \\
\text { X20 lors Mononucléose } \\
\text { X10 Si non diagnostiqué }\end{array}$ & Interaction virus- aérosols & 107 \\
\hline Sinusite & $\begin{array}{l}\text { Evolution saisonnière décalée par } \\
\text { rapport aux poussées de SEP }\end{array}$ & Aérosols irritants & 108 \\
\hline $\begin{array}{l}\text { Pollution de } \\
\text { l'air }\end{array}$ & $\begin{array}{l}\text { Exacerbation et Augmentation des } \\
\text { poussées OR }=1.44 \text { (femmes) }\end{array}$ & & 109 \\
\hline Tabagisme & $\mathrm{RR}=1.48$ & & 110 \\
\hline $\begin{array}{l}\text { Exposition aux } \\
\text { solvants }\end{array}$ & $\mathrm{OR}=1.53$ & & 111 \\
\hline $\begin{array}{l}\text { Porteur de } \\
\text { HLADRB1*15 }\end{array}$ & RR =X43 (gène de susceptibilité) & Lors d'exposition aux solvants & 112 \\
\hline
\end{tabular}


Figure 1

La dimension temporo-spatiale des facteurs de risques d'origine environnementale, représentation graphique en fonction du temps et de l'espace (adapté du Livret Environnement, Académie des sciences, 20.02.2002, p:35 on line accès le 30.11. 2003)

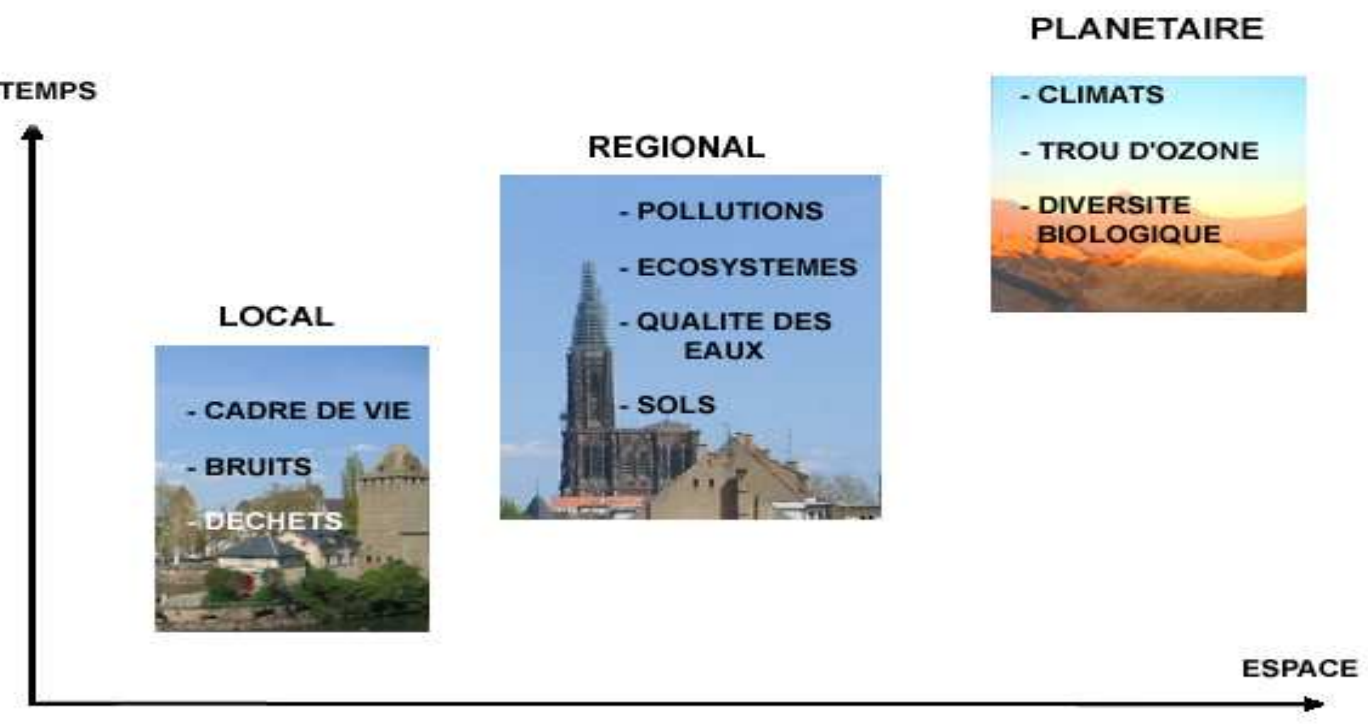

